\title{
ER network homeostasis is critical for plant endosome streaming and endocytosis
}

\author{
Giovanni Stefano ${ }^{1}$, Luciana Renna ${ }^{1}$, YaShiuan Lai ${ }^{1}$, Erin Slabaugh ${ }^{1}$, Nicole Mannino ${ }^{1}$, Rafael A Buono ${ }^{2,3}$, \\ Marisa S Otegui ${ }^{2,3,4}$, Federica Brandizzi ${ }^{1}$ \\ ${ }^{1}$ MSU-DOE Plant Research Lab and Plant Biology Department, Michigan State University, East Lansing, MI, USA; \\ ${ }^{2}$ Department of Botany, University of Wisconsin-Madison, Madison, WI, USA; ${ }^{3}$ R. M. Bock Laboratories Cell and Molecular \\ Biology, University of Wisconsin-Madison, Madison, WI, USA; ${ }^{4}$ Department of Genetics, University of Wisconsin-Madison, \\ Madison, WI, USA
}

Eukaryotic cells internalize cargo at the plasma membrane via endocytosis, a vital process that is accomplished through a complex network of endosomal organelles. In mammalian cells, the ER is in close association with endosomes and regulates their fission. Nonetheless, the physiological role of such interaction on endocytosis is yet unexplored. Here, we probed the existence of ER-endosome association in plant cells and assayed its physiological role in endocytosis. Through live-cell imaging and electron microscopy studies, we established that endosomes are extensively associated with the plant ER, supporting conservation of interaction between heterotypic organelles in evolutionarily distant kingdoms. Furthermore, by analyzing ER-endosome dynamics in genetic backgrounds with defects in ER structure and movement, we also established that the ER network integrity is necessary for homeostasis of the distribution and streaming of various endosome populations as well as for efficient endocytosis. These results support a novel model that endocytosis homeostasis depends on a spatiotemporal control of the endosome dynamics dictated by the ER membrane network.

Keywords: Arabidopsis; endocytosis; endoplasmic reticulum; organelle streaming

Cell Discovery (2015) 1, 15033; doi:10.1038/celldisc.2015.33; published online 17 November 2015

\section{Introduction}

Endocytosis is an essential process in eukaryotes. It mediates the recycling of membranes, cell surface receptors and ion channels, and is crucially important for the control of the lipid and protein composition of the plasma membrane. Through regulation of either recycling or degradation of plasma membrane receptors, this transport step also contributes to the perception of external stimuli $[1,2]$. Endocytosis of cargo is thought to be vesicle mediated and leads to a class of organelles loosely defined as endosomes. These are membranous compartments that function as critical sorting stations for membrane traffic, protein targeting and maturation. The early endosomes (EEs) are the first to receive endocytic cargo [3]. In plant cells, the

Correspondence: Federica Brandizzi

Tel: +1-517-353-7872; Fax: +1-517-353-9168;

E-mail: fb@msu.edu

Received 26 July 2015; accepted 20 September 2015
Trans-Golgi Network (TGN) is the compartment where endocytic cargo accumulates first, and is thus regarded as the plant $\mathrm{EE}$ equivalent. It is widely believed that secretory and endocytic routes merge at the TGN/EE, and that endocytic cargo is either recycled back to the plasma membrane or sorted to the vacuole for degradation. The latter pathway passes via the multivesicular bodies (MVBs) or late endosomes (LEs)/prevacuolar compartments and late prevacuolar compartments $[4,5]$.

In plant cells, intracellular movement of organelles, including endosomes, relies largely on actin [6]. This is in contrast with mammalian cells where endosome movement depends on microtubules. Mammalian endosomes associate with endoplasmic reticulum (ER) tubules, which can wrap around them and regulate their fission [7]. Although it is yet unknown whether the ER-endosome association may have a role in endosomal function in protein traffic, these findings underscore a role of the ER in endosome biogenesis. In plant cells, it is yet unknown whether endosomes associate 
with the ER and whether the ER may exert a role in endosome biogenesis as well as endosome-driven processes related to membrane traffic homeostasis.

In plant cells, the ER can be found in association with the membranes of other organelles through points of contact that have been seen in electron microscopy [8] and live-cell imaging analyses [9]. However, the physiological relevance of such connections is unknown. Recent findings indicate that connection between the ER and other organelles is significant for organelle streaming, which in plant cells is markedly conspicuous. Although organelle streaming in plant cells depends predominantly on actin and associated myosin motors [10-13], it has been recently shown that reduced membrane fluidity and disruption of the ER network impedes the movement of other organelles [14]. It was postulated that this effect may be linked to alteration of the homeostasis of the contact points between ER and other organelles [14]. Intriguingly, the relevance of organelle movement in the context of organelle function is still an open question. For example, ER-Golgi membrane traffic can take place when the movement of Golgi stacks is inhibited by chemical disruption of actin [15]. However, whether ER-Golgi membrane traffic may be more efficient in intact cells compared with actin-depleted cells is yet to be established. Similarly, it is yet unknown if endosome streaming has a role in endosome-dependent processes, such as endocytosis.

In this work, we addressed the fundamental questions on whether the ER is in close association with endosomes in plant cells, and whether a functional correlation exists between organelle streaming and function. Through live-cell imaging and electron tomography analyses in the plant model species Arabidopsis thaliana, we established that endosomes entertain a close association with the ER in plant cells. Using established genetic models with disrupted ER network integrity, we also determined that the ER network is critical for maintaining homeostasis of the spatial distribution and dynamics of endosomes. Finally, using time-course experiments to trace internalization of the fluorescent endocytic probe, FM4-64, as well as analyses of auxin accumulation and distribution of auxin transporters, which obtain a polarized subcellular localization at the plasma membrane through selective endocytosis, we established that defects in the ER network integrity negatively influence endocytosis. Taken together, our results not only support that a close association of the ER with endosomes is conserved among eukaryotic kingdoms, they also offer new perspectives in the significance of organelle movement in plant cells by demonstrating a functional relationship between the dynamics of the ER and the role of endosomes in endocytic traffic.

\section{Results}

In plant cells, the ER and endosomes are spatially and temporally associated

To establish the spatial organization of endosomes in relation with the ER in plant cells, we performed time-course imaging analyses of leaf epidermal cells co-expressing the inert ER lumen marker (GFP-HDEL) [16] and fluorescent protein-tagged RabF2a or RabA1g. RabF2a is a marker of late endosomes/MVBs; RabAlg is a marker of post-TGN/ EEs [17, 18]. Using dual-color live-cell imaging, we observed that despite the continuous remodeling of the tubules and cisternal ER, the populations of endosomes labeled by the two Rab-fusion proteins appeared in extensive association with the ER network (Figures 1a and b, Supplementary Movie S1). Specifically, during time-lapse acquisitions we observed that the majority of the endosomes were closely associated with the ER network, while a small subpopulation of endosomes moved between ER tubules. To quantify the degree of association of the endosomes with ER over time, we followed the same approach adopted earlier in mammalian cells to track individual endosomes with respect to the ER network using live-cell imaging of cells co-expressing endosome and ER markers [7]. We tracked the movement of RabF2a- and RabA1g-labeled endosomes with respect to ER tubules for about $1 \mathrm{~min}$. We verified that continuous tracking with the ER was maintained for over $85 \%$ of RabF2apositive endosomes and for over $75 \%$ of the RabAlgpositive endosomes. Within the same time frame, the remaining endosomes were found in non-continuous association with the ER network (Figures 1c and d). These data indicate that the majority of the endosome populations visualized with RabF2a and RabA1g are extensively associated with the ER over time.

The association between ER and endosomes was further investigated by electron tomography (Figure 2). We obtained dual-axis tomograms of high-pressure frozen/freeze substituted Arabidopsis root epidermal cells and identified MVBs in close proximity to ER membranes. We analyzed 10 tomograms (300-nmthick) containing 15 MVBs. Physical ER-MVB contact was observed in two cases (Figures $2 \mathrm{a}$ and $\mathrm{a}^{\prime}$ ), whereas, other MVBs were just at a close distance with the ER (Figures $2 b$ and $b^{\prime}$ ). These results not only support the live-cell imaging observations that 

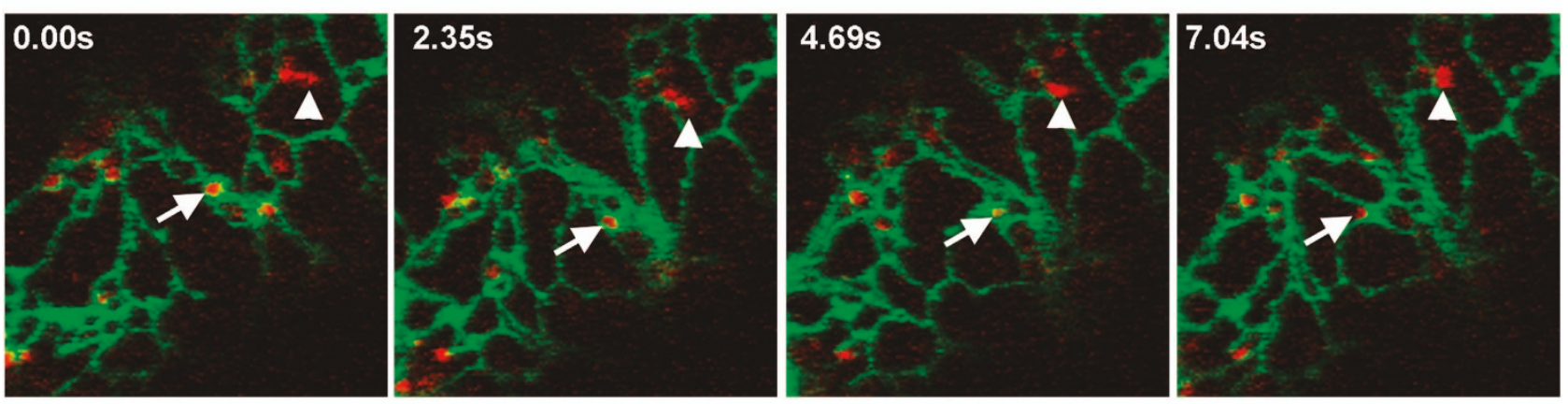

\section{b}
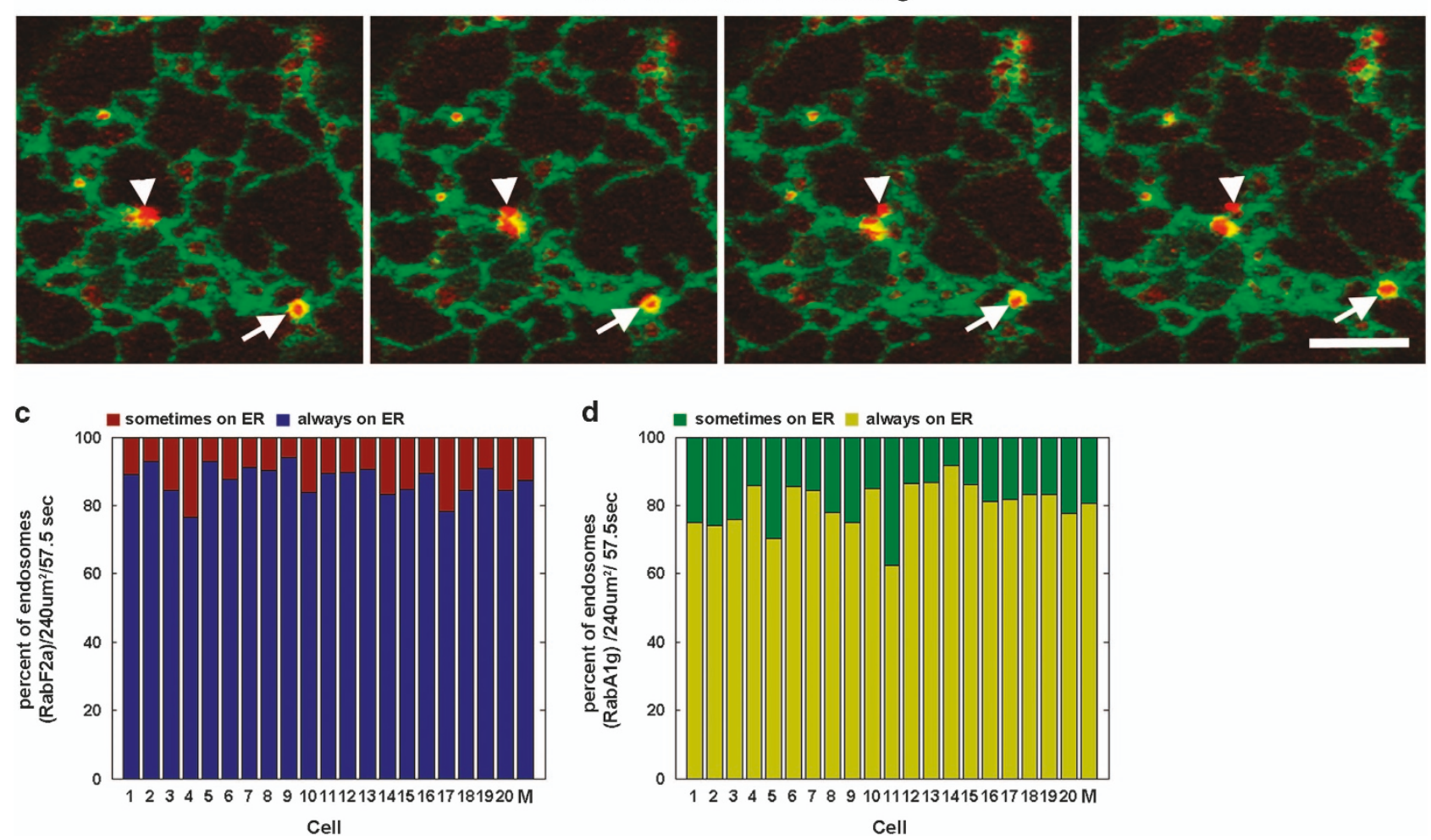

Figure $1 \mathrm{ER}$ and endosomes are associated in plant cells. (a, b) Time-lapse microscopy in the cortical region of a Nicotiana tabacum leaf epidermal cells expressing GFP-HDEL (ER lumen) and either YFP-RabF2a (late endosome/MVBs) or YFP-RabA1g (post-TGN/EE late endosome/pre-vacuolar compartment) reveals that the majority of the endosomes are associated with the ER membranes over time (arrow) or only for a limited time (arrowhead). (c, d) Quantification of the ER-endosome association over time in individual cells $(N=20)$ co-expressing GFP-HDEL and either YFP-RabF2a or YFP-RabA1g. The graph represents the percentage of endosomes that are continuously associated with the ER (always on ER) or are associated only for a limited number of frames (sometimes on ER) during the entire time-lapse sequence (57.5 s). The association was estimated for a total of 689 of RabF2a-labeled endosomes and 1165 RabA1g-labeled endosomes. The average of the percentages estimated for the 20 cells is also presented (M). Scale bars, $5 \mu \mathrm{m}$. ER, endoplasmic reticulum; MVBs, multivesicular bodies.

endosomes can be found close to the ER but also demonstrate that some of the endosomes may be in physical contact with the ER.

Together, our cell imaging analyses support that the plant ER entertains dynamic associations with the endosomes similar to the mammalian ER [7], underscoring unexpected but significant similarities between the spatial and temporal behavior of endosomal organelles and ER that are conserved across multicellular kingdoms. 

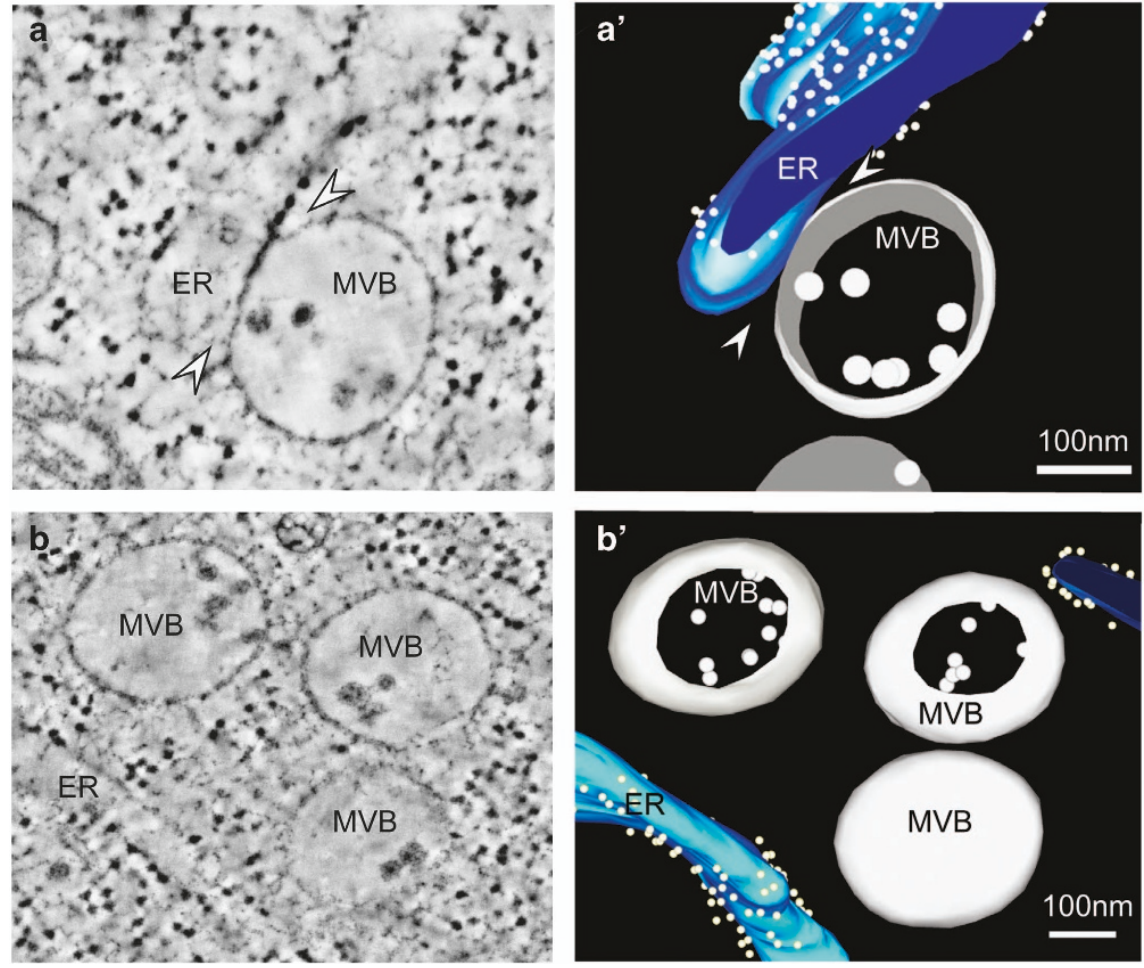

Figure 2 ER-endosome association revealed by electron tomography. Electron tomography slices (a, b) and corresponding three-dimensional reconstructions $\left(\mathbf{a}^{\prime}, \mathbf{b}^{\prime}\right)$ of $A$. thaliana root epidermal cells showing a close association between the ER membrane and multivesicular endosomes (MVBs; arrowheads). Scale bars, $100 \mathrm{~nm}$. ER, endoplasmic reticulum.

Disruption of ER network integrity affects endosome streaming

To establish whether endosome dynamics could be influenced by ER network integrity, we used the known ER membrane-modifier reticulon B3 (RTNLB3), which causes extensive remodeling of ER tubules when overexpressed $[19,20]$. Reticulons are a class of membrane proteins that facilitate membrane curvature [21]. In addition to ER network reshaping (Figure 3a), we determined that overexpression of RTNLB3 reduces the diffusion of ER membrane proteins (Supplementary Figure S1). On the basis of observed association of the ER with endosomes (Figures 1 and 2) and the modifying effect of RTNLB3 overexpression on the ER network, we predicted the occurrence of alterations in the dynamics of endosomes in cells overexpressing RTNLB3. To test this, we performed single-organelle streaming measurements in cells expressing the Rab markers alone or in combination with GFP-RTNLB3 (Figures 3b-d). Live-cell imaging analyses of 165 RabF2a-positive endosomes and 218 RabA1g-positive endosomes revealed that the velocity of endosomal streaming was significantly reduced in cells co-expressing GFP-RTNLB3 c ompared with cells expressing the Rab markers alone. Specifically, kymograph analyses [22] revealed that the average velocities of RabF2a-positive endosomes $\left(1.228 \pm 0.105 \mu \mathrm{m} \mathrm{s}^{-1}\right)$ were significantly reduced in cells overexpressing GFP-RTNLB3 $\left(0.605 \pm 0.055 \mu \mathrm{m} \mathrm{s}^{-1}\right.$; $P<0.0001$; Figure 3c). A similar trend was observed for the RabA1g-positive endosome population, as demonstrated by the evidence that the endosome velocity $\left(3.356 \pm 0.234 \mu \mathrm{m} \mathrm{s}^{-1}\right)$ was significantly reduced when GFP-RTNLB3 was co-expressed $\left(1.482 \pm 0.112 \mu \mathrm{m} \mathrm{s}^{-1} ; P<0.0001\right.$; Figure $\left.3 \mathrm{~d}\right)$. Intriguingly, these data indicate that the streaming velocity of RabF2a-positive endosomes is lower than that of RabA1g-positive endosomes. Indeed, colocalization analyses with the two Rab markers showed that the localization of the two endosome markers overlaps only partially (Supplementary Figure S2). Nonetheless, overexpression of the ER-membrane modifier GFP-RTNLB3 led to a similar reduction of streaming ( $50 \%$; Figures $3 \mathrm{c}$ and d) of both Rab-endosomal populations compared with cells with unmodified ER (Figures $3 \mathrm{c}$ and $\mathrm{d}$ ). On the basis of the results that streaming levels of both populations of endosomes are similarly disrupted by overexpression of the same 
a

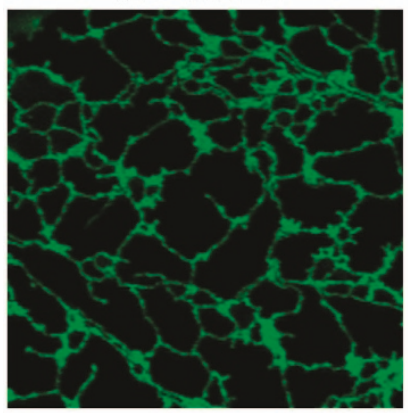

GFP-RTNLB3

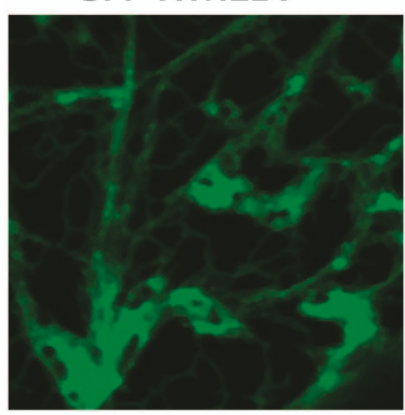

C

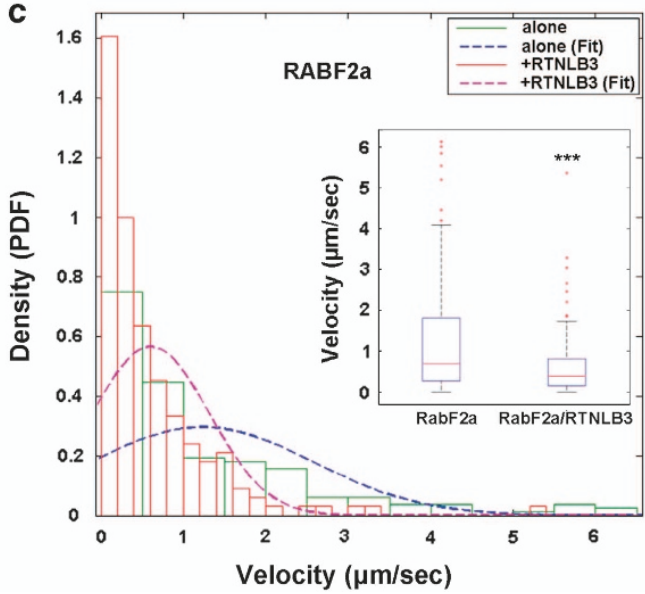

b GFP-RTNLB3

GFP-RTNLB3
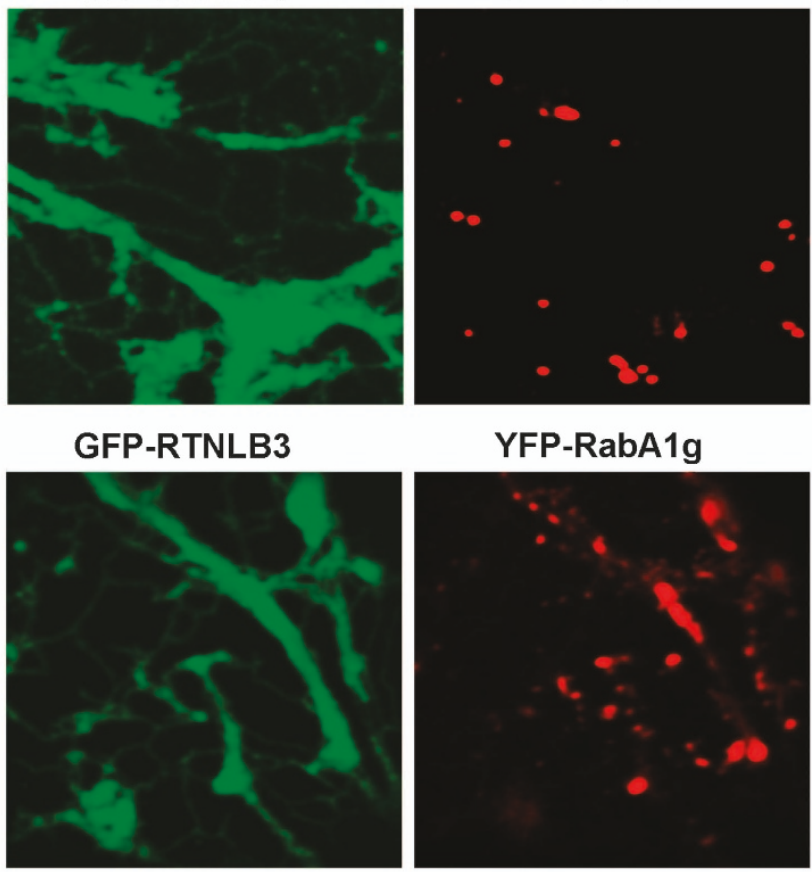

d

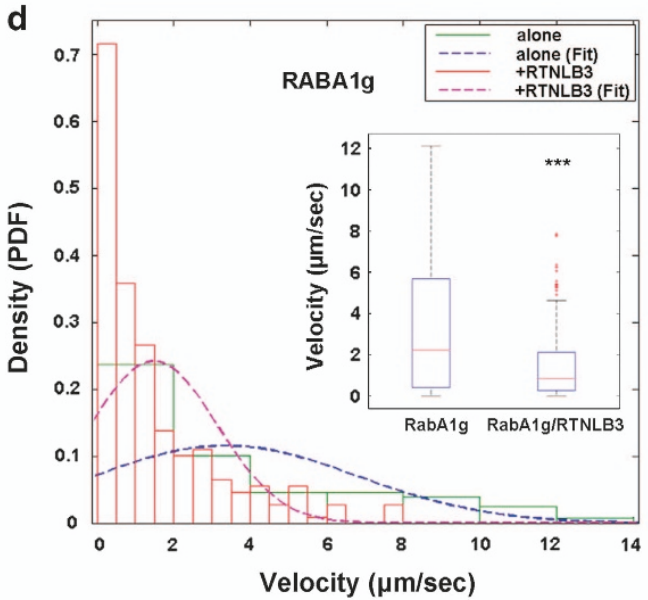

Figure 3 Disruption of ER network integrity compromises endosome velocity. (a, b) GFP-HDEL, GFP-RTNLB3, YFP-RabF2a and YFP-RabA1g were transiently expressed in tobacco leaf epidermal cells. GFP-HDEL reveals a typical ER network with tubules and cisternae; however, expression of GFP-RTNLB3 causes deformation of the ER network. Scale bars, $5 \mu \mathrm{m}$. (c, d) Streaming velocity measurements of 165 endosome organelles labeled by YFP-RabF2a (c) and 218 endosomes labeled by YFP-RabA1g (d) in tobacco epidermal cells in the absence (solid green lines) or in the presence of overexpressed GFP-RTNLB3 (solid red lines) were calculated from kymographs generated in ImageJ ( $1.49 \mathrm{~h})$. Endosome movement is expressed as histograms and probability density function (PDF) of the velocity distributions, which were fitted (blue and magenta dashed lines) using distribution tool (Matlab). Inset in $\mathbf{c}$ and $\mathbf{d}$ display the average velocity of the relative endosomes. Statistical analysis was performed using Student's $t$-test $\left({ }^{* * *} P<0.0001\right)$. ER, endoplasmic reticulum.

ER-modifying protein, we conclude that the dynamics of these endosomal populations is largely influenced by homeostasis of the ER membrane network.

To test whether the effect on the spatial distribution and dynamics of the endosomes could be a general phenotype linked to ER defects, we followed the distribution of endosomes in an Arabidopsis knockout mutant of ROOT HAIR DEFECTIVE 3 (rhd3), a critical ER-membrane associated dynamin-like protein that shapes the ER network [23], possibly through mediation of homotypic fusion [24, 25] of ER membranes. Compared with wild-type cells, in 
rhd3-depleted cells the ER network is arranged primarily into clusters of long unbranched tubules (Supplementary Figure S3) and the diffusion of proteins in the ER membrane is reduced [14, 23, 25, 26]. We hypothesized that if the ER integrity exerts a general effect on the spatial and dynamic organization of endosomes, as supported by the experiments with RTNLB3 (Figure 3), then the distribution and streaming velocity of these organelles would be also different in $r h d 3$ compared with wild type. To test this, we analyzed the distribution of an additional endosome marker, RabF2b, which labels late endosomes [27]. Indeed, live-cell imaging analyses revealed that in wild type, RabF2b-positive endosomes were uniformly distributed at the cell cortex; however, in the rhd3 mutant, we found partial partitioning of endosomes into clusters (Supplementary Figure S3B). The clusters were found also in the rhd3 mutant expressing the EE/TGN marker (AGD5) [28-30] (Supplementary Figure S4, panel A). To address whether the ER membrane clusters coincide with both EE/TGN and MVB/LE clusters (Supplementary Figure S4, panel B), we transiently overexpressed YFP-RHD3. This is known to induce aberrant ER sheets/clusters [31]. Indeed, cell imaging analyses revealed an overlap between the ER and EE/ TGN/MVB/LE clusters (Supplementary Figure S4). To test whether the streaming of endosomes was compromised in rhd3 compared with wild type, we tracked endosomes in time-lapse imaging analyses in wild type and rhd3 leaf cotyledon cells expressing YFP-RabF2b (Figure 4c). The analyses revealed that in wild-type cells, the average velocity of RabF2b-positive endosomes $\left(N_{\text {endosomes }}=221\right)$ was $2.554 \pm 0.139 \mu \mathrm{m} \mathrm{s}^{-1}$, which is significantly higher than that in rhd3 cells $\left(0.373 \pm 0.039 \mu \mathrm{m} \mathrm{s}^{-1} ; P<0.0001\right.$; Figures $4 \mathrm{~d}$ and e). These results indicate that disruption of the ER morphology due to the loss of RHD3 leads to defects in the spatial distribution and streaming velocity of endosomes. The demonstrated disruption of the dynamics of endosomes in cells that either overexpress RTNLB3 (Figure 3) or lack RHD3 (Figure 4) argues that the observed defects in endosome distribution and dynamics are attributable to general ER network defects, and supports that ER network integrity is required for optimal streaming of endosomal organelles in plant cells.

\section{ER morphology defects partially disrupt endocytosis}

We next addressed the question whether spatiotemporal anomalies in the distribution of endosomes could have an impact on cell physiology by analyzing endocytosis, which relies on endosomal function. As customary to study endocytosis homeostasis, we monitored the internalization of the endocytic tracer FM4-64 [4, 32-36] in a time-course experiment in wild type and rhd 3 root cells. Consistent with the previous observations in wild-type cells [37], FM4-64 fluorescence appeared at the PM and endosomal structures within $5 \mathrm{~min}$ of incubation and fluorescence internalization progressed over time (Figure 5a). However, compared with wild type, in rhd3 cells, the internalization of the dye resulted delayed. To quantify this phenomenon, we estimated the ratio between the FM4-64 fluorescence signal at the PM and cytoplasm [38]. We found that the PM/cytoplasm ratio was significantly lower in rhd3 compared with wild type, supporting a shift of fluorescence accumulation at the PM in rhd3 compared with the wild-type control. These results support the possibility that endocytosis rate is reduced in $r h d 3$ compared with wild type (Figures $5 \mathrm{a}-\mathrm{f}$ ). To validate this observation, we used brefeldin A (BFA), a fungal toxin that inhibits exocytic traffic from the endosomes to the PM but does not inhibit endocytic traffic from the PM to endosomes. In wild-type cells, BFA treatment generally leads to endosome aggregations, which are known as BFA bodies, therefore disruption of endocytosis homeostasis delays formation of such bodies [39]. Consistent with this notion and the preponderant accumulation of FM4-64 at the rhd3 PM compared with wild type (Figures $5 \mathrm{a}-\mathrm{f}$ ), BFA treatment led to a conspicuous appearance of BFA bodies in wild type but not in rhd3 (Figure $5 \mathrm{~g}$ and Supplementary Figure S5). To test whether the loss of RHD3 affected secretion, we monitored the distribution of a bulk-flow marker (SEC-RFP [40]) and a vacuolar marker (AFVY-RFP [41]) in wild type (Supplementary Figures S6A and B) and rhd3 cells during cell expansion (Supplementary Figures S6C and D). Changes in the export ability of the ER in the mutant would be translated into variations in the subcellular distribution of these markers compared with wild type; however, no substantial differences in the distribution of the markers were noted. Together, these results show that ER defects linked to abnormal availability of the ER-shaping protein such as RHD3 reduce endocytosis.

Cellular availability of the auxin transport machinery is reduced in rhd3 compared with wild type

We next aimed to probe the physiological consequences of reduced levels of endocytosis in rhd3. In plant cells, endocytosis is critical for the intracellular trafficking and polarity of the transporters 
a

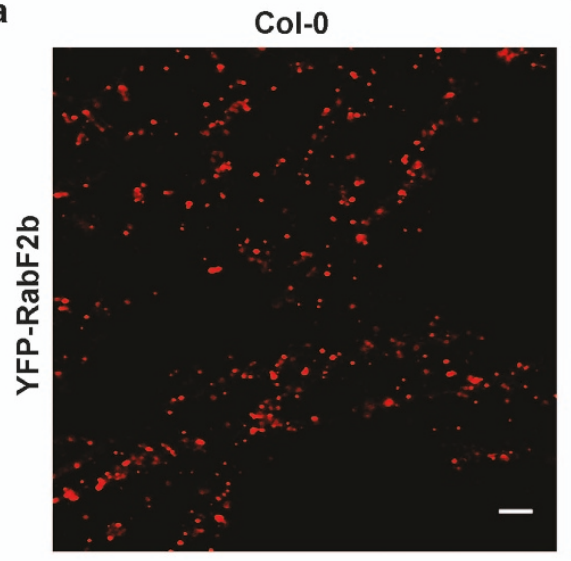

c

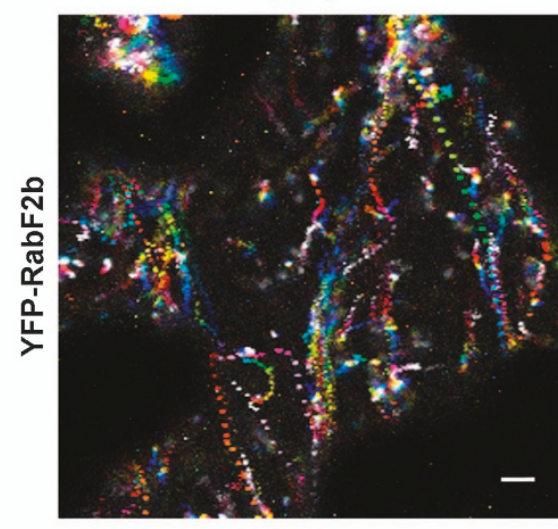

d

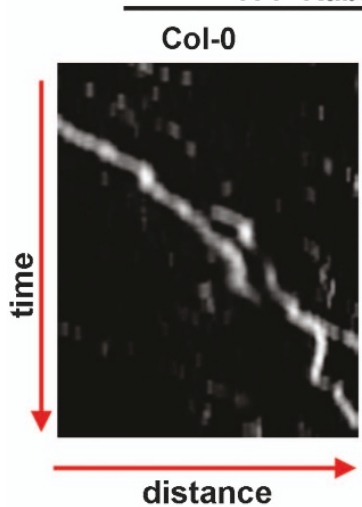

e

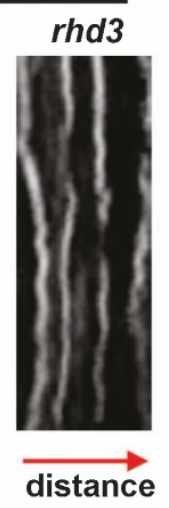

b

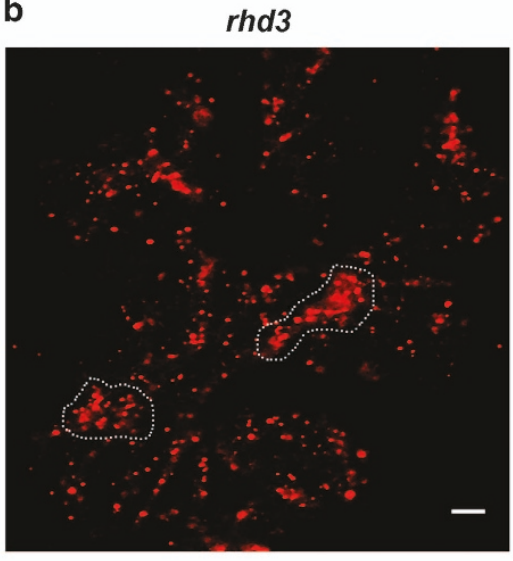

rhd3
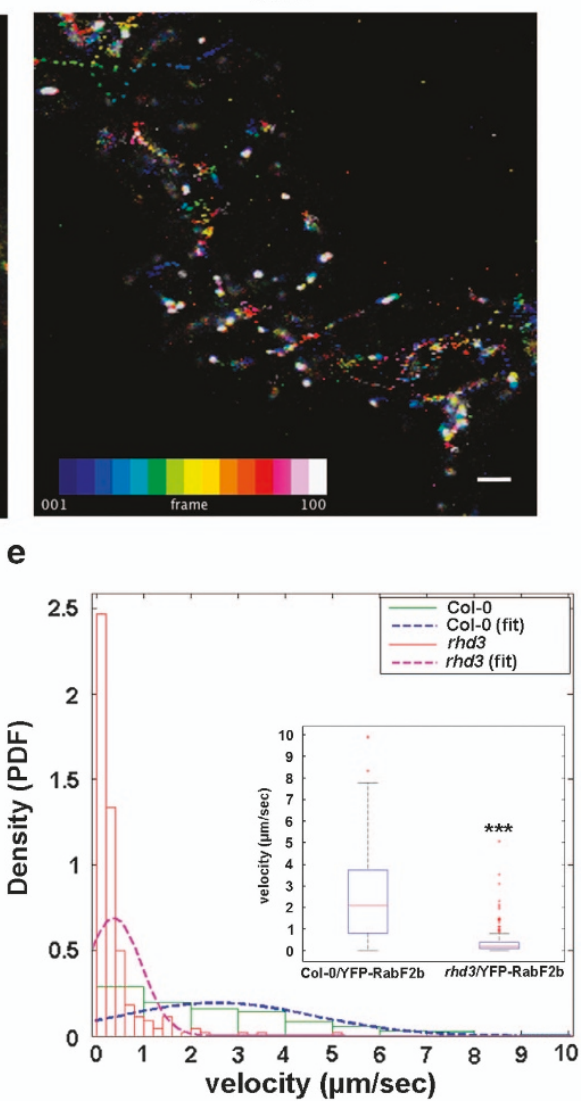

Figure 4 Spatiotemporal distribution of endosome is compromised in rhd3. (a, b) Confocal images of cotyledon epidermal cells in wild type (a; Col-0) and $r h d 3$ (b) (rhd3) expressing the endosome marker YFP-RabF2b, note the clustering of endosomes in rhd3 (circled by dotted line). (c) Intracellular streaming of endosome organelles in Col-0 and $r h d 3$ mutant presented as super-imposed frames of a time-lapse sequence $(25 \mathrm{~s})$. In each frame, organelles have been assigned a color, with dark red representing the position of organelles in initial frames and white indicating the position of the organelles in the last frame. Intermediated colors are presented at the bottom of the panel. Reduced streaming of endosomes in rhd 3 is evidenced by local superimposition of colors compared with wild type. (d). Representative kymographs for Col-0/YFP-RabF2b and rhd3/YFP-RabF2b. (e) Endosome movement of 221 YFP-RabF2b-labeled endosomes in Col-0 (red) and rhd3 (green) is expressed as histograms and probability density function (PDF) of the velocity distributions, which were fitted (blue and magenta dashed lines) using distribution tool (Matlab). Statistical analysis was performed using Student's $t$-test $\left.{ }^{* * *} P<0.0001\right)$. The inset plot shows the average velocity for endosomes in wild type and mutant cells. Scale bars, $5 \mu \mathrm{m}$. 


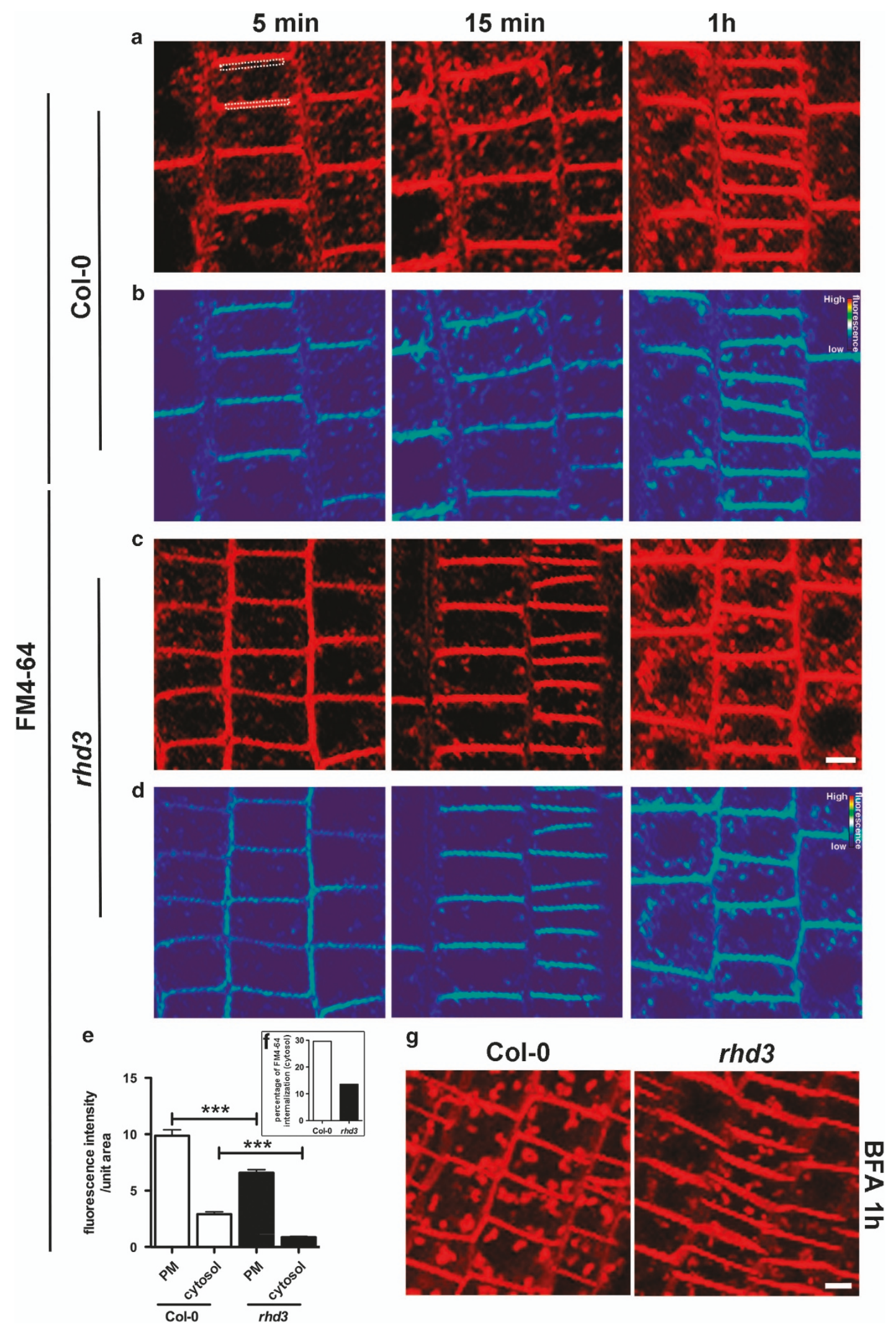


of the hormone auxin, a master regulator of plant growth [42, 43]. Application of exogenous auxin disrupts endocytosis, as demonstrated by a decrease in FM4-64 uptake in roots treated with auxin [39]. Furthermore, compared with wild type, yucca mutants, which are defective in auxin biosynthesis, are characterized by higher concentrations of endogenous auxin (IAA) and reduced FM4-64 internalization [39]. It is also known that high concentrations of auxin inhibit root growth [44]. Because rhd3 mutant shows both defect in endocytosis (Figure 5) and root elongation [23, 45], we hypothesized that endogenous levels of auxin and homeostasis of auxin transport machinery would be compromised in this mutant. To test this hypothesis, we first applied IAA on wild type and $r h d 3$, and established that primary root growth was inhibited in both backgrounds compared with the respective controls (Supplementary Figure S7). Intriguingly, the root growth of untreated rhd3 seedlings was comparable to that of IAA-treated wild-type seedlings and exogenous IAA treatment led to lower root growth inhibition in rhd3 compared with wild type (Supplementary Figure S7). These results indicated that rhd3 is sensitive to auxin but also supported the possibility that rhd3 roots contain higher levels of endogenous auxin compared with wild-type roots. We tested this by analyzing the fluorescence intensity of a live-auxin reporter based on the fusion of an auxin-responsive promoter DR5 to a fluorescent protein [46, 47], which enables to assess auxin levels in wild-type and $r h d 3$ roots. Quantitative confocal microscopy analyses of the fluorescence of the auxin reporters in the root apex indicated that the auxin levels were significantly higher in $r h d 3$ compared with wild type (Figure 6). These results support our initial hypothesis that the levels of auxin are compromised in rhd3. Because application of exogenous auxin reduces the intracellular levels of PIN-auxin transporters [48], and because we found higher auxin levels in $r h d 3$ compared with wild type (Figure 6), we next aimed to test whether the auxin transport machinery could be affected in rhd3 roots. To test this, we analyzed the abundance of established fluorescent reporters of components of the auxin efflux (PIN1, PIN5) and influx (LAX3, AUX1) machinery in rhd3 and wild-type roots. Quantitative confocal microscopy analyses showed that the fluorescence levels in transgenic lines expressing either Pro $_{\text {pin } 1}:: P I N 1-G F P$

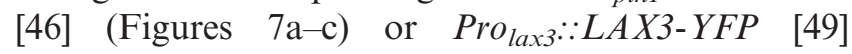
(Figures $7 \mathrm{~d}-\mathrm{f}$ ) were significantly reduced compared with wild type. An overall decrease in the fluorescence levels could be also observed for the auxin transporters 35S::PIN5-GFP [50] or 35S::AUX1-YFP [51] (Supplementary Figure S8). Together, these results indicate that loss of the ER shaping RHD3 leads to increased levels of auxin and disruption of the homeostasis of transport machinery, which consequently perturbs the homeostatic distribution of these proteins at the plasma membrane.

\section{Discussion}

Structural integrity of organelles is a requirement for their optimal function. Indeed, ER architecture defects lead to severe subcellular phenotypes, which in plants and mammals have been associated to severe growth and developmental phenotypes [14, 52-54], but the underlying mechanisms are unknown. Recent literature, however, points toward a new paradigm that disruption of the morphological integrity of the ER also affects the positioning of other organelles both in mammalian and plant cells [14, 55]. Nonetheless, the extent to which disruption of the ER network homeostasis influences the function of other organelles is yet to be defined. Our results support that ER network integrity is a requirement for the positioning and dynamics of endosomes and for endocytosis. These results not only provide evidence that association of endosomes and ER is conserved in plant and mammalian cells, they also demonstrate that endocytosis depends on ER network integrity. We propose that disruption of the ER tubular network affects the homeostasis of the subcellular distribution and dynamics of ER-associated endosomes, which is required for efficient endocytosis. By demonstrating that loss of ER structure leads also to disruption of

Figure 5 Endocytosis is delayed in rhd3. (a, c) Time course ( $5 \mathrm{~min}, 15 \mathrm{~min}, 1 \mathrm{~h}$ ) to follow uptake of the endocytic tracer FM4-64 in Col-0 and $r h d 3$ seedlings. (b, d) Fluorescence signal intensities represented as heat map facilitates visualization of levels of FM4-64 uptake in the two genetic backgrounds. (e) The graph shows a quantification of the FM4-64 signals in the root epidermis (fluorescence signal, after 5 min of FM4-64 treatment, was measured at plasma membrane and cytosol as indicated by the white dashed rectangles in a). Statistical analysis was performed using Student's $t$-test $\left({ }^{* \star *} P<0.0001\right)$. (f) The graph represents the percentage of FM4-64 internalized looking at the cytosolic area. (g) BFA bodies observed in the maximal projection of confocal images of 6 -day-old Arabidopsis seedlings (Col-0 and rhd3 root epidermal cells) pulsed with FM4-64 marker and treated with $100 \mu \mathrm{m} \mathrm{BFA} \mathrm{for} 1 \mathrm{~h}$. BFA, brefeldin A. 
a

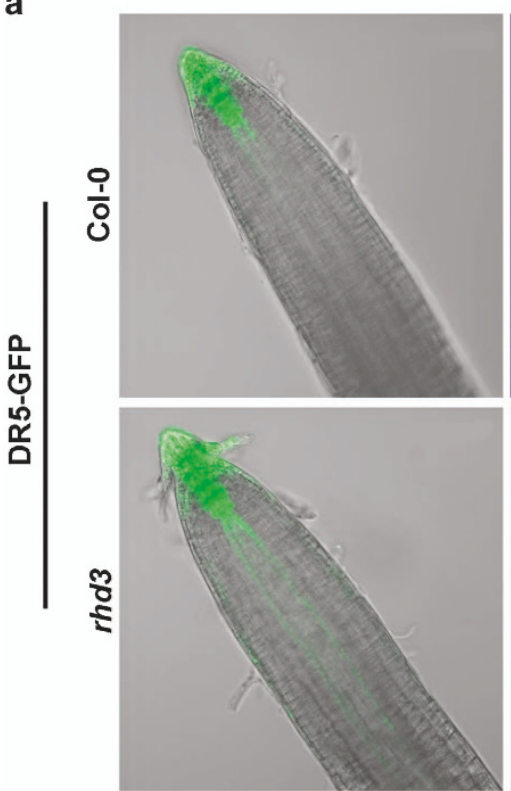

d

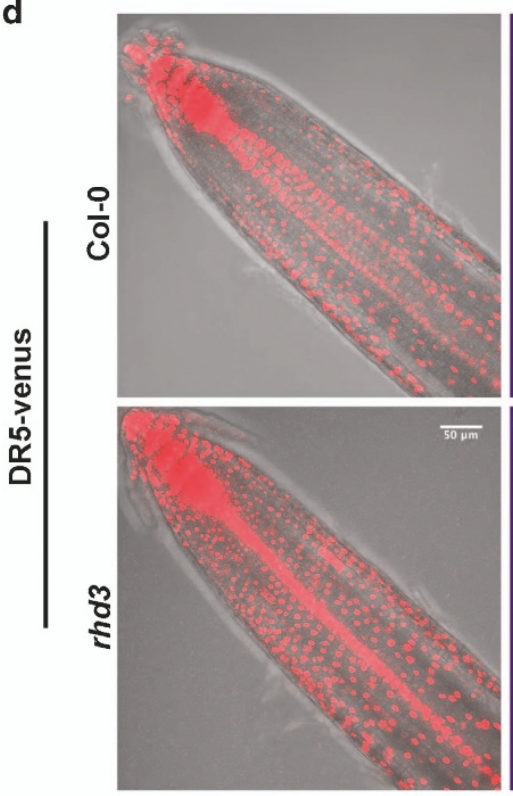

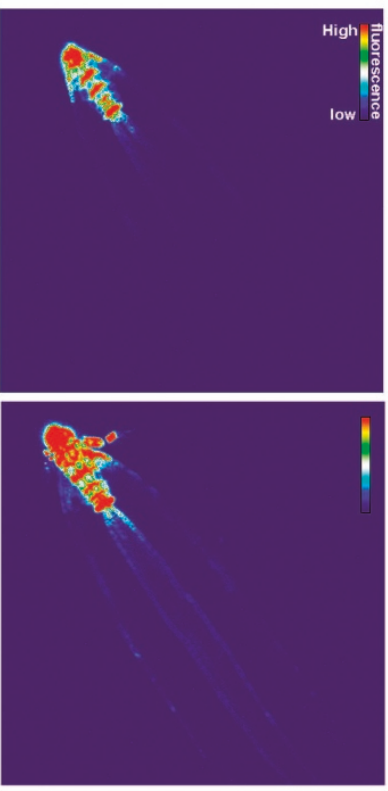

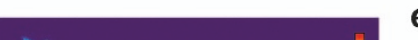

b
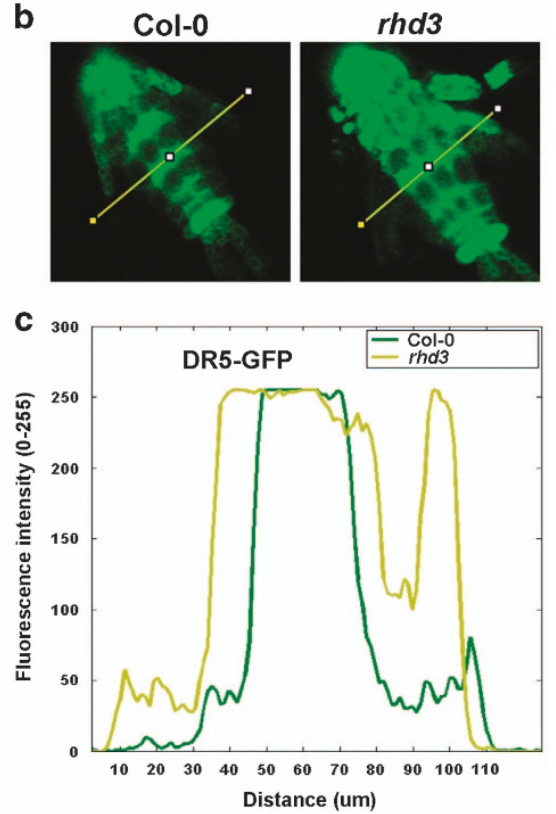

e Col-0

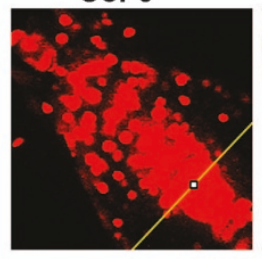

rhd3
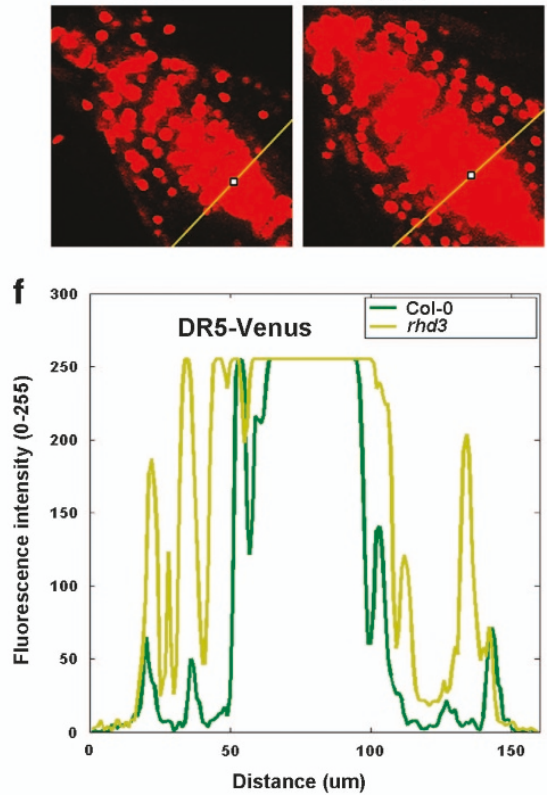

Figure 6 Auxin abundance. (a, d) Representative confocal image of DR5-GFP and DR5-VENUS (GFP or VENUS driven by the synthetic auxin-response promoter DR5 that reflects relative auxin levels) roots (7DAG) in wild type (Col-0) and rhd3 showing an enlarged area with levels of auxin in the longitudinal axis rhd3 roots compared with wild type. (b) Confocal image of DR5-GFP root at the apex and (c) quantification of the fluorescence in the graph. (e) Confocal image of DR5-Venus root at the apex and (f) quantification of the fluorescence in the graph. The images displayed are representative of at least three independent experiments with $>10$ seedlings examined each experiment. Scale bar, $50 \mu \mathrm{m}$.

physiological levels of auxin transporters and aberrant intracellular accumulation of auxin, we propose that ER-induced defects in endocytosis may contribute to plant growth phenotypes of mutants of the ER-shaping protein RHD3.
The association of the ER with endosomes is conserved in eukaryotes

The ER is a pervasive organelle and arguably the organelle with the largest membrane area. Recent studies in mammalian cells have provided evidence for 


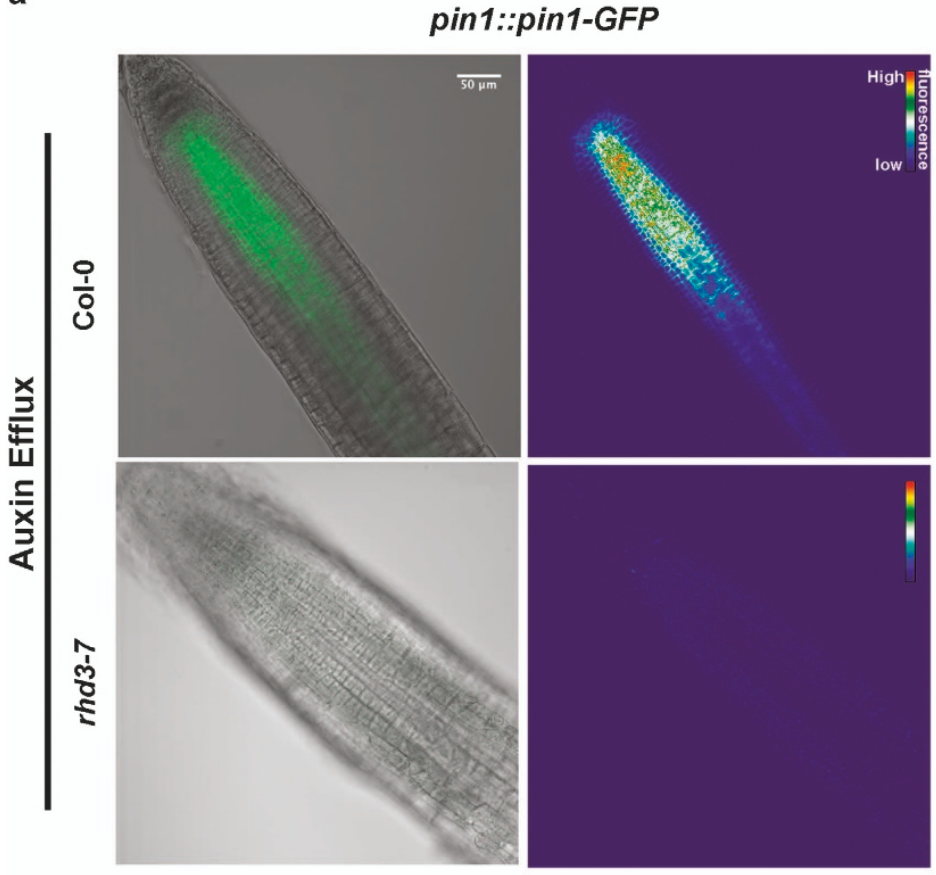

d

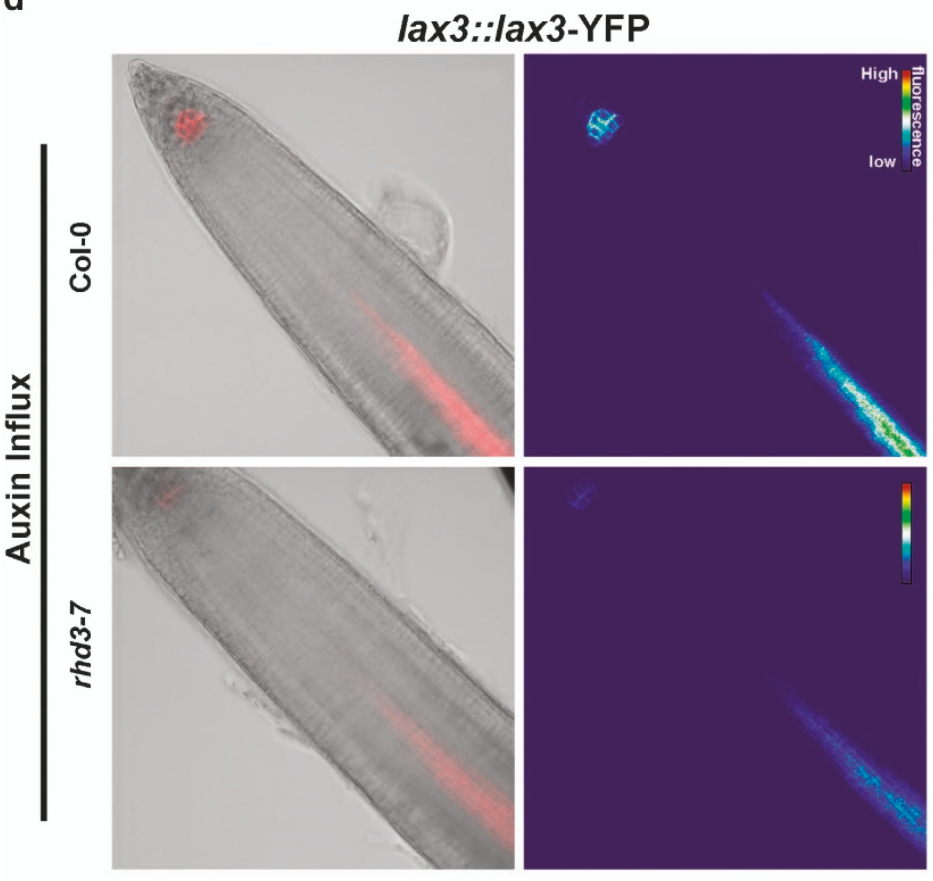

b

Col-0/
pin1::pin1-GFP

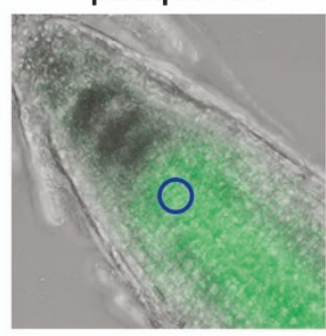

c

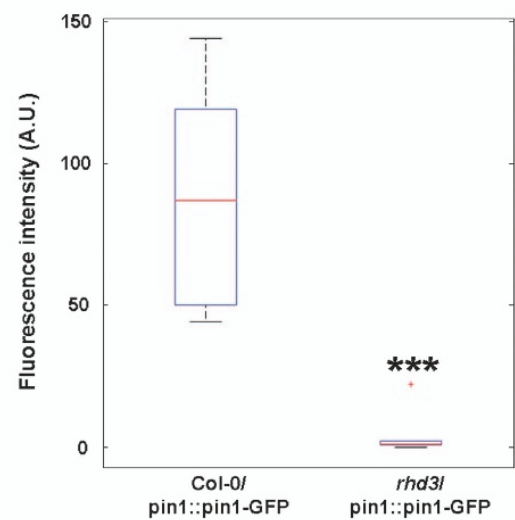

e

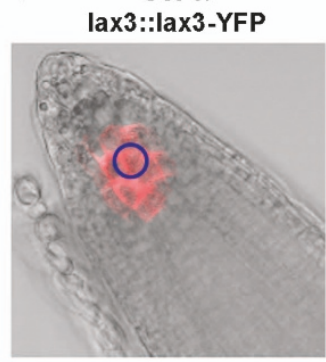

rhd3/
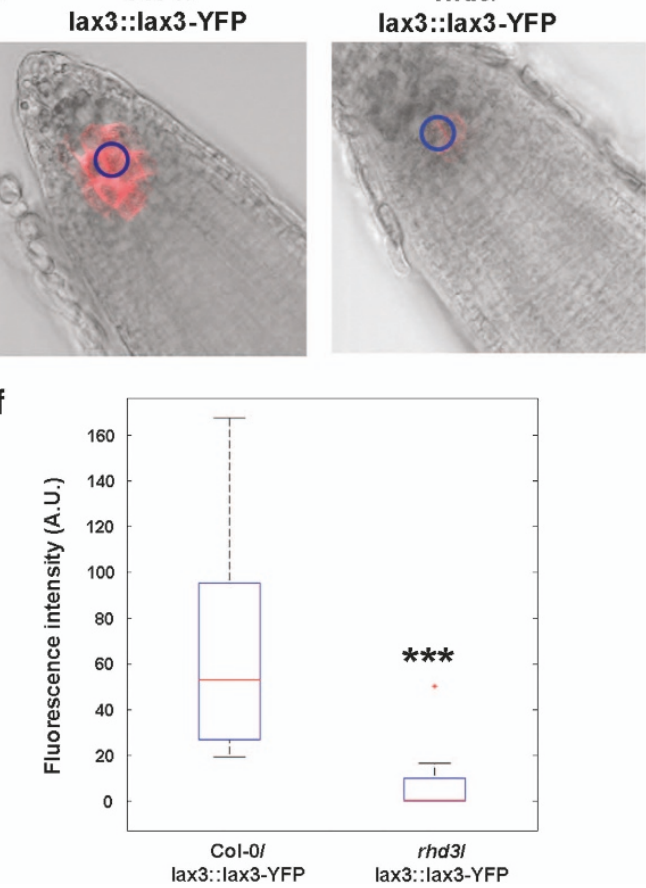

Figure 7 The levels of the efflux and influx machinery are compromised in rhd3. (a) Increased endogenous levels of auxin lead to a shut down of the PIN1-GFP expression in rhd3 root compared with wild type (Col-0). (b). Close-up view of PIN1::PIN1-GFP fluorescence at the root apex shows a signal intensity differences between Col-0 and $r h d 3$. (c) PIN1::PIN1-GFP signal changes can be observed in the graph $(n=8)$. (d) LAX3::LAX3-YFP levels in Col-0 and rhd3 root. (e) Close-up view of LAX3::LAX3-YFP fluorescence at the root apex shows signal intensity differences between Col-0 and $r h d 3$. (f) LAX3-YFP signal changes can be observed in the graph $(n=12)$. Statistical analysis was performed using Student's $t$-test $\left({ }^{* * *} P<0.0001\right)$. Scale bar, $50 \mu \mathrm{m}$. 
the existence of contact sites between endosomal organelles and ER membranes with a closer association between ER and late endosomes compared with the early endosomes [7]. Molecular candidates responsible for the connection between late endosomes and ER, like STARD3, STARD3NL and VAP, have been recently reported [56]. The identification of these proteins and of possible molecular bridges involving ORP1L-VAP (oxysterol-binding protein-VAMPassociated protein) and EGFR-PTP1B (epidermal growth factor receptor-protein tyrosine phosphatase) [57] supports that the association of the ER with endosomes may most likely exert specific functions. In this work, we have demonstrated that the ER is in extensive association with endosomes over time in plant cells. In marked contrast with mammalian cells, in plant cells, the ER is in close association with Golgi stacks, denoting a unique organization of the plant early secretory pathway. The evidence provided in this work that in plant cells endosomes are in association with the ER, similar to mammalian cells, poses that, differently from the organization of the Golgi and the $\mathrm{ER}$, there is conservation of the association of the ER with post-Golgi organelles in eukaryotes.

\section{Endosomal streaming depends on ER integrity}

It has been recently shown that defects in plant ER structure and membrane composition influence the streaming of other organelles [58]. Specifically, lack of RHD3 correlates with reduced streaming of the ER, Golgi, peroxisomes and mitochondria, independently from actin cytoskeleton integrity [14]. These findings delineated a model wherein the ER exerts a force on the movement of other organelles that are in contact with the ER [14, 59]. In this work, we have demonstrated that loss of RHD3 has a profound effect also on the streaming of endosomes. The findings that disruption of ER network homeostasis due to overexpression of RTNLB3, an ER-shaping reticulon, has a similar effect on endosome streaming compared with loss of RHD3 argues that the observed effect is not specific to RHD3 or RTNLB3 but rather to a general loss of ER network integrity.

In this work, through photobleaching experiments we have established that overexpression of reticulon leads to changes in the ER membrane protein diffusion, as established by reduction of the mobility of a bulk ER membrane marker in conditions of RTNLB3 overexpression and RHD3 loss compared with wild type. It is possible that overabundance of reticulon may impede the homeostasis of distribution of other ER membrane proteins, which could explain alteration in the fluorescence recovery after photobleaching of a bulk membrane marker. It was similarly hypothesized that loss of RHD3 may alter the distribution of reticulons or other ER-shaping proteins and therefore change the overall ER membrane protein diffusion [14]. We speculate that if ER-endosome contacts are maintained by membrane-anchored protein tethers, the observed changes in endosome movement in the rhd3 loss-of-function mutant as well as reticulonoverexpressing cells could be due, at least partially, to altered diffusion of the ER-endosome tethers in the ER membrane. Although this hypothesis waits for experimental validation, the evidence that membrane protein tethers between the ER and other organelles exist in non-plant organisms [59] lends partial support to our hypothesis.

It has been shown that although the ER is a contributing force to organelle streaming in plant cells, the streaming velocity can be markedly different among the various organelle types [14]. In this work, we have observed that the streaming velocity of RabF2a-positive endosomes $\left(1.228 \pm 0.105 \mu \mathrm{m} \mathrm{s}^{-1}\right)$ was significantly lower than that of RabAlg-positive endosomes $\left(3.356 \pm 0.234 \mu \mathrm{m} \mathrm{s}^{-1}\right)$ in cells with intact ER. The evidence provided in our work that the distribution of the two Rab markers do not completely overlap supports marked diversification of the streaming requirements for the various endosomal populations in plant cells. The observation that loss of ER integrity halves the average velocity of these populations of organelles supports that, although the ER is a common driving force for the streaming of both populations, the residual motor force may be exerted by diverse motor proteins that contribute to the organelle streaming with different propelling forces.

\section{Defects in endosome distribution due to ER network disruption compromise endocytosis, auxin homeostasis and organ growth}

Live-cell imaging analyses in mammalian cells have shown that disruption of ER structure and tubule movement due to overexpression of human Reticulon 4a (Rtn4a) reduces the rate of endosome fission [55], supporting that the ER has a role in the homeostasis of endosome biogenesis. However, the consequences of loss of endosomal homeostasis due to disruption of ER integrity in the physiology of cells and of the whole organism are yet unknown. In plant cells, endosomes are highly mobile, as demonstrated earlier [6] and in this work, but it is yet to be established whether their movement has any functional relevance for the cell and organism. In our live-cell imaging analyses with the 
endocytic tracer FM4-64 we have found that alteration of the distribution and streaming of the endosomes is associated with a delay in FM4-64 uptake, which indicates that endocytosis is partially compromised. These conclusions are further supported by the evidence that the plasma membrane accumulation of auxin transporters, which depends on endocytosis [60], is altered in the rhd3 mutant with defects in ER morphology. These results pose that a dysregulation in the streaming of the endosomes contribute to a dysfunction in their normal activities. The underlying causes of this phenotype are yet unknown. Endocytic and secretory cargo pass through the TGN/EE and MVBs/LEs; the latter endosome type may contain PM proteins destined to degradation and may originate from TGN/EE through maturation processes [3,61]. Although it is possible that defects in the ER network may impede endosome maturation, it is also possible that they may compromise endosome fission and therefore reduce the number of available endosomes for endocytosis homeostasis. However, we cannot exclude that in the ER-defective mutants, the auxin defects may be also partially due to a hampered distribution of ER-localized auxin transporters due to defects in ER linked to RHD3 loss of function.

Plant growth and development depends on hormone homeostasis [43]. Small plant stature with marked reduction of root growth is a striking phenotype of all the RHD3 mutants characterized to date [23-25]. Defects in auxin transport due to altered endocytosis are known to alter cellular auxin homeostasis [62, 63]. Furthermore, increase in auxin levels leads to a decrease in PIN protein abundance [48] and reduction in root elongation [64]. The evidence provided in our work that in the rhd3 loss-of-function mutant the subcellular distribution of critical components of the auxin transport machinery is compromised and that the cellular levels of auxin are more elevated compared with wild type are consistent with the hypothesis of a genetic interaction of RHD3 with the auxin pathway [65]. Because rhd3 shows severe defect in ER morphology and endosome dynamics, we propose that the association of endosomes with the ER is critical for homeostasis of endocytosis and consequently, for growth regulation in plants.

\section{Materials and Methods}

\section{Cloning, plant materials and growth conditions}

RTNLB3 (AT1G64090), RabF2a (AT5G45130) and RabA1g (AT3G15060) were cloned into either pVKH18En6 (RTNLB3) or pEarlyGateway104 (Rabs) plant expression vectors. Four-week-old Nicotiana tabacum (cv Petit Havana) greenhouse plants grown at $25^{\circ} \mathrm{C}$ were used for Agrobacterium tumefaciens (strain GV3101)-mediated transient expression [66]. The bacterial optical density $\left(\mathrm{OD}_{600}\right)$ used for plant transformation was 0.05 for tagged versions of GFP-HDEL, CNX, RTNLB3, RabF2a, RabA1g and CFP-AGD5 [15, 66], while for YFP-RHD3 it was 0.3. Homozygous transgenic Arabidopsis thaliana lines (Col-0 ecotype) expressing ER-YK were described previously [14]. DR5-GFP, DR5-Venus, Pin1::PIN1-GFP, LAX3::LAX3-YFP, 35S::PIN5-GFP, 35S::Aux1-YFP seeds were described earlier [47-50, 67, 68]. The transgenes were introgressed into the $r h d 3$ background through crosses to enable quantitative comparisons of the fluorescence intensity between wild type and $r h d 3$. For IAA treatment, we used gom8, a recessive missense mutation in the At3g13870 locus (RHD3), which is complemented by RHD3 expression [23]. rhd3 is a T-DNA insertion null allele of RHD3 (rhd3-7) [23, 69]. Seeds were surface sterilized and germinated at $21^{\circ} \mathrm{C}$ under $16 \mathrm{~h}$ light/ $8 \mathrm{~h}$ dark conditions.

Confocal laser scanning microscopy and image analyses

An inverted laser scanning confocal microscope Zeiss LSM510 META (http://www.zeiss.com/) was used for confocal analyses. The fluorescent proteins used in this work were GFP5 [70], Venus and EYFP. The markers used in this study were the ER lumenal marker, ER-YK [71], and the ER membrane markers: RTNLB3 and calnexin [72], fused to GFP. RabF2a, RabF2b and RabA1g tagged with fluorescent proteins were used as endosome markers [27, 35]. Imaging settings for single fluorochromes or combinations were as described previously $[40,73,74]$. To analyze the velocity of the endosomes, 23 timelapse sequences for RabF2a and RabA1g (50 frames per timelapse sequence), and 43 time-lapse sequences for RabF2b (100 frames for each time-lapse sequence) were recorded in the cortical region of cotyledon pavement cells. The time-lapse frames $(512 \times 512$ pixel or $256 \times 256$ resolution) were captured with $2 \mu \mathrm{m}$ pinhole diameter, $10 \%$ power of an argon $488 \mathrm{~nm}$ laser line, and four digital zoom using an EC Plan-Neofluar $\times 40 / 1.30$ oil M27 objective. Kimograph analysis was performed in post acquisition using ImageJ plugins (v1.49). Velocity values were calculated by averaging the velocity of each endosome in each time-lapse sequence; then mean values were calculated as the mean of the velocities estimated in the 23 or 43 time-lapse sequences. Velocity values were estimated by averaging data values of each time-lapse sequence for each sample. Statistical analysis was based on $t$-test for velocity measurement and for FRAP data. Photoshop (http://www.adobe.com/) was used for further image handling.

\section{FRAP analyses}

Leaf segments were incubated in latrunculin B $(25 \mu \mathrm{m})$, which is a potent disrupter of actin filaments [75], for $30 \mathrm{~min}$ before imaging, as described earlier [15]. For FRAP experiments, the ER in the cortical region of abaxial epidermal pavement cells of $N$. tabacum leaves was used. Ten prebleach scans were captured using settings for GFP with the 488-nm laser transmission set to $1-2 \%$ transmission before bleaching of a $3.6 \mu \mathrm{m}^{2}$ spot using 30 iteractions of a 488/514-nm laser lines set 
to $100 \%$ transmission. The number of bleach events captured was 66 for CNX-GFP, and 68 for GFP-RTNLB3. Normalized data were plotted using MatLab (www.mathworks.com), as described previously [28].

\section{Drug and dye treatments}

Six-day-old Arabidopsis seedlings were incubated in $0.5 \times$ MS medium with $2 \mu \mathrm{M}$ FM4-64 (500 $\mu \mathrm{M}$; stock DMSO) for $5 \mathrm{~min}$, washed three times at room temperature, mounted, and observed. For BFA treatment, the seedlings were first incubated for 5 min with $2 \mu \mathrm{M}$ FM4-64 then incubated $1 \mathrm{~h}$ in $100 \mu \mathrm{M}$ BFA solution. Confocal microscopy analyses of the FM4-64-stained seedlings were performed on epidermal cells of the root tip.

\section{Electron microscopy}

Root tips from 1-week-old Arabidopsis seedlings were high-pressure frozen in a Baltec HP010, freeze-substituted in $2 \% \mathrm{OsO}_{4}$ in acetone, and embedded in Epon resin as described previously [76]. For electron tomography, tilt series from 300 -nm-thick sections were collected between $-60^{\circ}$ and $+60^{\circ}$, every $1^{\circ}$ in a FEI Tecnai F30 electron microscope operating at $300 \mathrm{kV}$. Tomogram calculation and segmentation was done with the IMOD package [77].

\section{Conflict of Interest}

The authors declare no conflict of interest.

\section{Acknowledgements}

We acknowledge support by the Chemical Sciences, Geosciences and Biosciences Division, Office of Basic Energy Sciences, Office of Science, US Department of Energy (award number DE-FG02-91ER20021) and National Science Foundation (MCB1243792) and AgBioResearch to FB, and National Science Foundation MCB1157824 to MSO.

\section{References}

1 Dhonukshe P, Aniento F, Hwang I et al. Clathrin-mediated constitutive endocytosis of PIN auxin efflux carriers in Arabidopsis. Curr Biol 2007; 17: 520-527.

2 Taylor NG. A role for Arabidopsis dynamin related proteins DRP2A/B in endocytosis; DRP2 function is essential for plant growth. Plant Mol Biol 2011; 76: 117-129.

3 Viotti C, Bubeck J, Stierhof YD et al. Endocytic and secretory traffic in Arabidopsis merge in the trans-Golgi network/early endosome, an independent and highly dynamic organelle. Plant Cell 2010; 22: 1344-1357.

4 Tse YC, Mo B, Hillmer S et al. Identification of multivesicular bodies as prevacuolar compartments in Nicotiana tabacum BY-2 cells. Plant Cell 2004; 16: 672-693.

5 De Marcos Lousa C, Gershlick DC, Denecke J. Mechanisms and concepts paving the way towards a complete transport cycle of plant vacuolar sorting receptors. Plant Cell 2012; 24: 1714-1732.
6 Voigt B, Timmers AC, Samaj J et al. Actin-based motility of endosomes is linked to the polar tip growth of root hairs. Eur J Cell Biol 2005; 84: 609-621.

7 Friedman JR, Dibenedetto JR, West M, Rowland AA, Voeltz GK. Endoplasmic reticulum-endosome contact increases as endosomes traffic and mature. Mol Biol Cell 2013; 24: 1030-1040.

8 Staehelin LA. The plant ER: a dynamic organelle composed of a large number of discrete functional domains. Plant J 1997; 11: 1151-1165.

9 Wang P, Hawkins TJ, Richardson C et al. The plant cytoskeleton, NET3C, and VAP27 mediate the link between the plasma membrane and endoplasmic reticulum. Curr Biol 2014; 24: 1397-1405.

10 Ueda H, Yokota E, Kutsuna N et al. Myosin-dependent endoplasmic reticulum motility and $\mathrm{F}$-actin organization in plant cells. Proc Natl Acad Sci USA 2010; 107: 6894-6899.

11 Peremyslov VV, Prokhnevsky AI, Dolja VV. Class XI myosins are required for development, cell expansion, and F-Actin organization in Arabidopsis. Plant Cell 2010; 22: 1883-1897.

12 Sparkes I, Runions J, Hawes C, Griffing L. Movement and remodeling of the endoplasmic reticulum in nondividing cells of tobacco leaves. Plant Cell 2009; 21: 3937-3949.

13 Prokhnevsky AI, Peremyslov VV, Dolja VV. Overlapping functions of the four class XI myosins in Arabidopsis growth, root hair elongation, and organelle motility. Proc Natl Acad Sci USA 2008; 105: 19744-19749.

14 Stefano G, Renna L, Brandizzi F. The endoplasmic reticulum exerts control over organelle streaming during cell expansion. J Cell Sci 2014; 127: 947-953.

15 Brandizzi F, Snapp EL, Roberts AG, Lippincott-Schwartz J, Hawes C. Membrane protein transport between the endoplasmic reticulum and the Golgi in tobacco leaves is energy dependent but cytoskeleton independent: evidence from selective photobleaching. Plant Cell 2002; 14: 1293-1309.

16 Brandizzi F, Hanton S, DaSilva LL et al. ER quality control can lead to retrograde transport from the ER lumen to the cytosol and the nucleoplasm in plants. Plant $J$ 2003; 34: 269-281.

17 Rutherford S, Moore I. The Arabidopsis Rab GTPase family: another enigma variation. Curr Opin Plant Biol 2002; 5: 518-528.

18 Asaoka R, Uemura T, Ito $\mathrm{J}$ et al. Arabidopsis RABA1 GTPases are involved in transport between the trans-Golgi network and the plasma membrane, and are required for salinity stress tolerance. Plant J 2013; 73: 240-249.

19 Sparkes I, Tolley N, Aller I et al. Five Arabidopsis reticulon isoforms share endoplasmic reticulum location, topology, and membrane-shaping properties. Plant Cell 2010; 22: 1333-1343.

20 Tolley N, Sparkes IA, Hunter PR et al. Overexpression of a plant reticulon remodels the lumen of the cortical endoplasmic reticulum but does not perturb protein transport. Traffic 2008; 9: 94-102.

21 Voeltz GK, Prinz WA, Shibata Y, Rist JM, Rapoport TA. A class of membrane proteins shaping the tubular endoplasmic reticulum. Cell 2006; 124: 573-586. 
22 Tan K, Roberts AJ, Chonofsky M, Egan MJ, Reck-Peterson SL. A microscopy-based screen employing multiplex genome sequencing identifies cargo-specific requirements for dynein velocity. Mol Biol Cell 2014; 25 : 669-678.

23 Stefano G, Renna L, Moss T, McNew JA, Brandizzi F. In Arabidopsis, the spatial and dynamic organization of the endoplasmic reticulum and Golgi apparatus is influenced by the integrity of the C-terminal domain of RHD3, a nonessential GTPase. Plant J 2012; 69: 957-966.

24 Zhang M, Wu F, Shi J et al. RHD3 family of dynamin-like GTPases mediates homotypic endoplasmic reticulum fusion and is essential for Arabidopsis development. Plant Physiol 2013; 163: 713-720.

25 Chen J, Stefano G, Brandizzi F, Zheng H. Arabidopsis RHD3 mediates the generation of the tubular ER network and is required for Golgi distribution and motility in plant cells. J Cell Sci 2011; 124: 2241-2252.

26 Stefano G, Brandizzi F. Unique and conserved features of the plant ER-shaping GTPase RHD3. Cellular Logist 2014; 4: e28217.

27 Nielsen E, Cheung AY, Ueda T. The regulatory RAB and ARF GTPases for vesicular trafficking. Plant Physiol 2008; 147: 1516-1526.

28 Stefano G, Renna L, Rossi M et al. AGD5 is a GTPaseactivating protein at the trans-Golgi network. Plant J 2010; 64: 790-799.

29 Sauer M, Delgadillo MO, Zouhar J et al. MTV1 and MTV4 encode plant-specific ENTH and ARF GAP proteins that mediate clathrin-dependent trafficking of vacuolar cargo from the trans-Golgi network. Plant Cell 2013; 25: 2217-2235

30 Liljegren SJ, Leslie ME, Darnielle L et al. Regulation of membrane trafficking and organ separation by the NEVERSHED ARF-GAP protein. Development 2009; 136: 1909-1918.

31 Zheng H, Chen J. Emerging aspects of ER organization in root hair tip growth: lessons from RHD3 and Atlastin. Plant Signal Behav 2011; 6: 1710-1713.

32 Ueda T, Uemura T, Sato MH, Nakano A. Functional differentiation of endosomes in Arabidopsis cells. Plant $J$ 2004; 40: 783-789.

33 Bolte S, Talbot C, Boutte Y, Catrice O, Read ND, Satiat-Jeunemaitre B. FM-dyes as experimental probes for dissecting vesicle trafficking in living plant cells. $J$ Micros 2004; 214: 159-173.

34 Dettmer J, Hong-Hermesdorf A, Stierhof YD, Schumacher K. Vacuolar H+-ATPase activity is required for endocytic and secretory trafficking in Arabidopsis. Plant Cell 2006; 18: 715-730.

35 Chow CM, Neto H, Foucart C, Moore I. Rab-A2 and Rab-A3 GTPases define a trans-golgi endosomal membrane domain in Arabidopsis that contributes substantially to the cell plate. Plant Cell 2008; 20: 101-123.

36 Jelinkova A, Malinska K, Simon S et al. Probing plant membranes with FM dyes: tracking, dragging or blocking? Plant J 2010; 61: 883-892.
37 Bach L, Gissot L, Marion J et al. Very-long-chain fatty acids are required for cell plate formation during cytokinesis in Arabidopsis thaliana. J Cell Sci 2011; 124: 3223-3234.

38 Boisson-Dernier A, Lituiev DS, Nestorova A, Franck CM, Thirugnanarajah S, Grossniklaus U. ANXUR receptorlike kinases coordinate cell wall integrity with growth at the pollen tube tip via NADPH oxidases. PLoS Biol 2013; 11: e1001719.

39 Paciorek T, Zazimalova E, Ruthardt N et al. Auxin inhibits endocytosis and promotes its own efflux from cells. Nature 2005; 435: 1251-1256.

40 Faso C, Chen YN, Tamura K et al. A missense mutation in the Arabidopsis COPII coat protein Sec24A induces the formation of clusters of the endoplasmic reticulum and Golgi apparatus. Plant Cell 2009; 21: 3655-3671.

41 Hunter PR, Craddock CP, Di Benedetto S, Roberts LM, Frigerio L. Fluorescent reporter proteins for the tonoplast and the vacuolar lumen identify a single vacuolar compartment in Arabidopsis cells. Plant Physiol 2007; 145: 1371-1382.

42 Jaillais Y, Chory J. Unraveling the paradoxes of plant hormone signaling integration. Nat Struct Mol Biol 2010; 17: $642-645$.

43 Ljung K. Auxin metabolism and homeostasis during plant development. Development 2013; 140: 943-950.

44 Evans M, Ishikawa H, Estelle M. Responses of Arabidopsis roots to auxin studied with high temporal resolution: comparison of wild type and auxin-response mutants. Planta 1994; 194: 215-222.

45 Wang HY, Lockwood SK, Hoeltzel MF, Schiefelbein JW. The ROOT HAIR DEFECTIVE3 gene encodes an evolutionarily conserved protein with GTP-binding motifs and is required for regulated cell enlargement in Arabidopsis. Genes Dev 1997; 11: 799-811.

46 Benkova E, Michniewicz M, Sauer M et al. Local, effluxdependent auxin gradients as a common module for plant organ formation. Cell 2003; 115: 591-602.

47 Brunoud G, Wells DM, Oliva M et al. A novel sensor to map auxin response and distribution at high spatiotemporal resolution. Nature 2012; 482: 103-106.

48 Vieten A, Vanneste S, Wisniewska $\mathbf{J}$ et al. Functional redundancy of PIN proteins is accompanied by auxindependent cross-regulation of PIN expression. Development 2005; 132: 4521-4531.

49 Swarup K, Benkova E, Swarup R et al. The auxin influx carrier LAX3 promotes lateral root emergence. Nat Cell Biol 2008; 10: 946-954.

50 Barbez E, Lankova M, Parezova M et al. Single-cell-based system to monitor carrier driven cellular auxin homeostasis. BMC Plant Biol 2013; 13: 20.

51 Swarup R, Kargul J, Marchant A et al. Structure-function analysis of the presumptive Arabidopsis auxin permease AUX1. Plant Cell 2004; 16: 3069-3083.

52 Audhya A, Desai A, Oegema K. A role for Rab5 in structuring the endoplasmic reticulum. J Cell Biol 2007; 178: 43-56.

53 Chen J, Stefano G, Brandizzi F, Zheng H. Arabidopsis RHD3 mediates the generation of the tubular ER network 
and is required for Golgi distribution and motility in plant cells. J Cell Sci 2011; 124: 2241-2252.

54 Park SH, Zhu PP, Parker RL, Blackstone C. Hereditary spastic paraplegia proteins REEP1, spastin, and atlastin-1 coordinate microtubule interactions with the tubular ER network. J Clin Invest 2010; 120: 1097-1110.

55 Rowland AA, Chitwood PJ, Phillips MJ, Voeltz GK. ER contact sites define the position and timing of endosome fission. Cell 2014; 159: 1027-1041.

56 Alpy F, Rousseau A, Schwab Y et al. STARD3 or STARD3NL and VAP form a novel molecular tether between late endosomes and the ER. J Cell Sci 2013; 126: $5500-5512$.

57 Rocha N, Kuijl C, van der Kant R et al. Cholesterol sensor ORP1L contacts the ER protein VAP to control Rab7RILP-p150 Glued and late endosome positioning. $J$ Cell Biol 2009; 185: 1209-1225.

58 Stefano G, Renna L, Brandizzi F. The endoplasmic reticulum exerts control over organelle streaming during cell expansion. J Cell Sci 2014; 127: 947-953.

59 Stefano G, Hawes C, Brandizzi F. ER - the key to the highway. Curr Opin Plant Biol 2014; 22C: 30-38.

60 Kitakura S, Vanneste S, Robert S et al. Clathrin mediates endocytosis and polar distribution of PIN auxin transporters in Arabidopsis. Plant Cell 2011; 23: 1920-1931.

61 Scheuring D, Viotti C, Kruger F et al. Multivesicular bodies mature from the trans-Golgi network/early endosome in Arabidopsis. Plant Cell 2011; 23: 3463-3481.

62 Ambrose C, Ruan Y, Gardiner J et al. CLASP interacts with sorting nexin 1 to link microtubules and auxin transport via PIN2 recycling in Arabidopsis thaliana. Dev Cell 2013; 24: 649-659.

$63 \mathrm{Li} \mathrm{G}$, Liang W, Zhang X et al. Rice actin-binding protein RMD is a key link in the auxin-actin regulatory loop that controls cell growth. Proc Natl Acad Sci USA 2014; 111: 10377-10382.

64 Eliasson L, Bertell G, Bolander E. Inhibitory action of auxin on root elongation not mediated by ethylene. Plant Physiol 1989; 91: 310-314.

65 Wang HY, Lee MM, Schiefelbein JW. Regulation of the cell expansion gene RHD3 during Arabidopsis development. Plant Physiol 2002; 129: 638-649.

66 Batoko H, Zheng HQ, Hawes C, Moore I. A rab1 GTPase is required for transport between the endoplasmic reticulum and golgi apparatus and for normal golgi movement in plants. Plant Cell 2000; 12: 2201-2218.

67 Kleine-Vehn J, Dhonukshe P, Swarup R, Bennett M, Friml J. Subcellular trafficking of the Arabidopsis auxin influx carrier AUX1 uses a novel pathway distinct from PIN1. Plant Cell 2006; 18: 3171-3181.
68 Ottenschlager I, Wolff $\mathrm{P}$, Wolverton $\mathrm{C}$ et al. Gravityregulated differential auxin transport from columella to lateral root cap cells. Proc Natl Acad Sci USA 2003; 100: 2987-2991.

69 Alonso JM, Stepanova AN, Leisse TJ et al. Genome-wide insertional mutagenesis of Arabidopsis thaliana. Science 2003; 301: 653-657.

70 Haseloff J, Siemering KR, Prasher DC, Hodge S. Removal of a cryptic intron and subcellular localization of green fluorescent protein are required to mark transgenic Arabidopsis plants brightly. Proc Natl Acad Sci USA 1997; 94: 2122-2127.

71 Nelson BK, Cai X, Nebenfuhr A. A multicolored set of in vivo organelle markers for co-localization studies in Arabidopsis and other plants. Plant $J$ 2007; 51: 1126-1136.

72 Irons SL, Evans DE, Brandizzi F. The first 238 amino acids of the human lamin B receptor are targeted to the nuclear envelope in plants. $J$ Exp Bot 2003; 54: 943-950.

73 Brandizzi F, Snapp EL, Roberts AG, Lippincott-Schwartz J, Hawes C. Membrane protein transport between the endoplasmic reticulum and the Golgi in tobacco leaves is energy dependent but cytoskeleton independent: evidence from selective photobleaching. Plant Cell 2002; 14: 1293-1309.

74 Hanton SL, Chatre L, Renna L, Matheson LA, Brandizzi F. De novo formation of plant endoplasmic reticulum export sites is membrane cargo induced and signal mediated. Plant Physiol 2007; 143: 1640-1650.

75 Nebenfuhr A, Gallagher LA, Dunahay TG et al. Stop-andgo movements of plant Golgi stacks are mediated by the acto-myosin system. Plant Physiol 1999; 121: $1127-1142$.

76 Chanoca A, Otegui MS. Immunogold labeling and electron tomography of plant endosomes. Methods Mol Biol 2014; 1209: 63-80.

77 Kremer JR, Mastronarde DN, McIntosh JR. Computer visualization of three-dimensional image data using IMOD. J Struct Biol 1996; 116: 71-76.

(Supplementary Information is linked to the online version of the paper on the Cell Discovery website.)

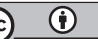

This work is licensed under a Creative Commons Attribution 4.0 International License. The images or other third party material in this article are included in the article's Creative Commons license, unless indicated otherwise in the credit line; if the material is not included under the Creative Commons license, users will need to obtain permission from the license holder to reproduce the material. To view a copy of this license, visit http://creativecommons.org/licenses/by/4.0/ 